Article

\title{
Using Free Adoptions to Reduce Crowding and Euthanasia at Cat Shelters: An Australian Case Study
}

\author{
Heather M. Crawford, Joseph B. Fontaine and Michael C. Calver* \\ Environmental and Conservation Sciences Cluster, School of Veterinary and Life Sciences, Murdoch University, \\ Murdoch 6150, Australia; crawfh01@gmail.com (H.M.C.); j.fontaine@murdoch.edu.au (J.B.F.) \\ * Correspondence: m.calver@murdoch.edu.au; Tel.:+61-8-360-6000
}

Received: 11 October 2017; Accepted: 22 November 2017; Published: 4 December 2017

Simple Summary: Waiving adoption fees to encourage adoptions and reduce euthanasias of healthy adult cats in crowded shelters is controversial because of concerns that people attracted to free adoptions may be less responsible owners. An extremely busy kitten season in 2015 left no shelter or foster vacancies for incoming cats at Western Australia's largest cat shelter. Instead of euthanasing healthy cats, the shelter held a three day adoption-drive where cats $\geq 1$ year were free. Public response to the event was extremely high (weekly adoptions increased $>5$-fold). Post-adoption surveys were carried out and results compared with surveys of cat adopters who paid normal-fees during non-promotional periods. No differences were found between free and normal-fee adopter demographics, cat demographics, cat fate post-adoption, incidence of medical and behavioural issues, and likelihood of attaching collars, registering with local councils or allowing cats to roam freely. Mixed-media promotion of the adoption-drive attracted more first-time adopters compared with normal-fee adopters. Overall, we found no evidence for adverse outcomes associated with free adoptions. Animal shelters should not be dissuaded from occasional free adoption-drives during overflow periods.

\begin{abstract}
Many healthy adult cats are euthanised annually in shelters, and novel approaches are required to reduce euthanasia rates. Waiving adoption fees is one such approach. However, concerns that less responsible owners will be attracted to free events persist among welfare groups. We evaluated evidence for differences in cat fate, health, and adherence to husbandry legislation via a case-study of a free adoption-drive for cats $\geq 1$ year at a Western Australian shelter. Post-adoption outcomes were compared between free adopters and a control group of normal-fee adopters. The free adoption-drive rehomed 137 cats, increasing average weekly adoptions by $533 \%$. First-time adopters were a significantly larger portion of the free cohort, as a result of mixed-media promotions. Both adopter groups selected cats of similar age; sex and pelage. Post-adoption, both groups retained $>90 \%$ cats, reporting near identical incidences of medical and behavioural problems. Adopters did not differ in legislative compliance regarding fitting collars, registering cats, or allowing cats to roam. The shelter reported satisfaction with the adoption-drive, because in addition to relieving crowding of healthy adults, adoption of full-fee kittens increased $381 \%$. Overall, we found no evidence for adverse outcomes associated with free adoptions. Shelters should not be dissuaded from occasional free adoption-drives during overflow periods.
\end{abstract}

Keywords: Australia; cat; euthanasia; free adoption; husbandry; legislation; promotion; shelter

\section{Introduction}

Globally, the domestic cat (Felis sylvestris catus) is a popular companion animal, with $18 \%$ of UK households [1], 26\% of European households [2], 35\% of USA [3], 44\% of New Zealand [4] and $29 \%$ of Australian households [5] owning cats. Popularity, and a continuous reproductive cycle with 
induced ovulation ( 2-3 litters per year depending on age, health, number of daylight hours and latitude [6-12]), inevitably leads to uncontrolled breeding and abandonment of cats at welfare shelters. For example, from mid-2015 to mid-2016, cats comprised 40\% of the total number of animals processed nationally by the Australian Royal Society for Prevention of Cruelty to Animals [13]; higher than dogs at $33 \%$. The total number of cats euthanized over the same period was nearly three times that of dogs (16,205 cats vs. 5872 dogs). A similar situation occurs in the United States, where a survey of American shelters revealed for the fiscal year 2008-2009 the highest euthanasia rates for cats in a shelter was 71\%, compared to $35 \%$ for dogs in the same facility [14].

While many cats entering shelters are euthanised due to illness, disease or behavioural issues [15, 16], healthy adult cats are also euthanised due to shelter crowding or financial strain [14,16]. A few countries prohibit this by law (e.g., Brazil [17] and Czech Republic [16]), but that may create other problems such as crowding or refusal to admit animals to a full shelter. Compared with adult cats, kittens may be more expensive to care for. In addition to requiring multiple vaccinations and more frequent worming than adult cats, there is potential extra nutritional care and associated labour (e.g., feeding every $4 \mathrm{~h}$ for kittens that have not been weaned). However, kittens are easier to rehome and can attract higher adoption fees $[15,18]$, so they may receive preferential treatment and housing over healthy or resident adult cats during times of crowding. High, on-going euthanasia rates also precipitate stress and poor morale of shelter staff, veterinarians and volunteers $[19,20]$. It is, therefore, in the best interests of both cats and staff that shelters minimise euthanasia rates.

To avoid euthanasia of older but otherwise adoptable cats, shelters may employ a range of strategies including refusing admission of cats during busy periods (e.g., 'kitten seasons'), and outsourcing cats to other charities, management groups or foster caregivers. Alternatively, shelters may opt to hold adoption-drives wherein typical adoption fees are reduced or waived to achieve higher adult rehoming rates and relieve capacity pressures [21,22].

Waiving adoption fees is the most controversial option, because of concerns that people attracted to low-cost or free adoptions may be less responsible owners who subsequently neglect or rehome cats, or use them for nefarious purposes (e.g., kitten farming, dog baiting). However, in Maine, United States, whether or not adopters paid for their cat had no influence on their emotional attachment to their pet, or their opinion of the shelter [21]. Similarly, in Queensland, Australia, adopters who paid a discounted fee of $\$ 20$ or regular fee $\geq \$ 99$ for adult cats, did not differ in their retention of the cat at time of survey. Both groups also had similar self-rated attachment to- and satisfaction with, their cats. They did not differ in their intention to keep the cat, willingness to adopt from the shelter in the future and in caretaking behaviours shown towards the cat (e.g., frequency of petting, worming etc.). However, the authors acknowledged imprecision in the effect estimates in their statistical analyses [22].

Given the limited number of studies on the topics of discounted or free adoption-drives and concern over the strength of inference from prior studies, we sought to add further evidence to the literature via a case study of a free-adoption drive at a Western Australian cat shelter that focused on characteristics of adopters (age, gender, history of prior adoptions, source of information about the shelter), fate of cats (retained, deceased, returned to shelter, rehomed privately), compliance of owners with relevant legislation (Western Australian Cat Act 2011), and owner experiences with shelters. We also compared the data from the free-adoption drive with control data from normal-fee adoptions from the same shelter to see if paying adopters differed from free adopters on these points. Although the study was primarily descriptive, based on the limited available literature, we predicted that:

1. Cat demographics (age, sex and pelage) and fate (e.g., dead, returned) would not differ between the free and normal-fee adopters;

2. Human demographics (e.g., gender, distance from shelter, average income) would not differ among free and normal-fee adopters; and

3. Both groups would be equally compliant with the requirements of relevant Western Australian legislation. 
Evidence consistent with these predictions would provide further support for shelter managers to proceed with greater confidence to use free adoptions in order to relieve accommodation and resource pressures during peak periods. In contrast, lack of support for our predictions would suggest reconsideration of free adoptions as a viable strategy for decreasing euthanasia.

\section{Materials and Methods}

\subsection{Study Context}

The Cat Haven shelter in Perth, Western Australia (WA), is the state's largest recipient of feral, stray and surrendered cats, processing $>4000$ cats per year. These cats come mainly from across the 30 local government jurisdictions in Perth ( 1.95 million people in 2016 [23]), but small numbers do come from outside the Perth metropolitan area. In accordance with the Australian Cat Action Plan for cat management ([24] developed by Getting2Zero), the Cat Haven has a long-term goal of zero euthanasia, excepting those animals which will never be rehomeable because of factors such as terminal illness or lack of socialisation. During 2014 and 2015 weekly euthanasias fluctuated seasonally, but for adult and mature cats were mainly in the range 2-22 and 0-21 for kittens. Despite having year-round discounted prices for cats over one year of age, an extremely busy 2014-2015 kitten season left the shelter stretched beyond capacity. A decision was made to trial a three-day adoption-drive where adult cats ( $\geq 1$ year) would be given away for free.

The Cat Haven desexes and microchips all cats prior to adoption, so we focused on other facets of animal husbandry likely to vary among owners: fitting collars, council registration, and whether or not cats were permitted to roam freely. The Cat Haven provides all cat adopters with a booklet of information on basic cat behaviours and tips for assimilating them into new homes; possible illnesses, and worming and vaccination routines. The Cat Haven has an adoption process that includes verifying photographic identification and address, and a right to refuse service to uncooperative customers.

The legal context within Western Australia changed in 2011 with the passage of the Cat Act [25], which requires WA cat owners to ensure that by six months of age all cats are desexed, microchipped, wearing a collar and registered with the owner's local municipal council. While the Cat Act (2011) does not mandate restricting cats to their owners' property, we take the provision of fines, trapping and euthanasia of nuisance animals as an implication that owners should not permit their cats to roam. As such, the Cat Haven distributes leaflets on the WA Cat Act regulations and requirements for responsible cat ownership to adopters, and sends a notice of adoption to the adopter's local council so that cat legislation may be monitored and enforced.

\subsection{Data Collection}

In February 2015, a week-long advertising campaign promoted the 'free adult cat adoption-drive' via Cat Haven's website, social media pages, local newspapers, radio and television-news programmes. Free cats included 'Adults' (1-7 years old), and 'Matures' ( $>7$ years). Kittens remained available for adoption at their regular year-round prices. Over the three day long-weekend (28th February-2nd March), 137 free cats were adopted (118 adult and 19 mature). Of these, the 100 cat adopters who had provided useable contact details were approached 6-12 months after adoption, using emails, postal letters, and telephone interviews ( $n=50,25$, and 25 respectively) depending on personal details voluntarily provided at time of adoption.

A larger post-adoption study using the same survey was carried out between December 2014 and May 2015. A further 120 randomly-selected people (as the largest manageable sample size for our team) who adopted adult or mature cats for a 'normal-fee' (\$50-\$180) during this period, were contacted and their responses compared with those of free cat adopters. Subjects were selected using random number protocols. Normal-fee adopters were contacted 3-18 months post-adoption using emails, postal letters, and telephone interviews ( $n=45,50$, and 25 respectively). 
To gauge the Cat Haven's perception of the event, we invited the CEO to comment on experiences over the weekend, and asked specifically about euthanasia and financial implications of the free adoption-drive. In order to assess how rates of adult/mature cat adoption over the free weekend differed from normal weeks of the year in 2015, the total weekly number of adoptions was plotted using the ggplot2 package within $\mathrm{R}$ version $3.1 .1[26,27]$. The total weekly number of kitten adoptions was also plotted for comparison, as were adoptions over the course of 2014. Euthanasia data for the years 2014 and 2015 were provided by the Cat Haven as a context for assessing the potential effect of the free weekend on euthanasia rates.

\subsection{Questionnaire Design and Owner Demographics}

Surveys for owners adopting free or normal-fee cats were identical (Appendix A Table A1). Surveys focused on the husbandry and fate of adopted cats, the gender of the adopters, their adoption history with cat shelters and means of learning about the Cat Haven shelter. Demographic information for adopter suburbs was obtained from the Australian Bureau of Statistics ([23], see results), in preference to producing a longer, less user-friendly questionnaire if this information was requested directly from adopters.

\subsection{Statistical Analyses}

Differences in response rates between free and normal-fee adopters were assessed using the interface for comparing proportions in the online resource VassarStats [28]. Basic demographics of cats adopted for free or for payment were tabulated, noting sex, age in months, pelage, and fate after adoption. Similarly, the demographics of owners adopting cats (free vs. paid) were tabulated, noting gender, adoption history, knowledge of Cat Haven, and a range of characteristics of their suburb (e.g., distance from shelter, population density, mean salary [23]) as potential indicators of socio-economic status. Based on the people who responded, this resulted in a data matrix of 151 cats and 141 unique households. All subsequent statistical analyses of these variables used lme4 and ggplot2 packages within $R$ version 3.1.1 [26,27,29].

To assess associations between adoption fee (free vs. paid), cat attributes (age category, sex, pelage, adoption fate), and adopter attributes (gender, adoption history with shelters, means of learning about Cat Haven), count data were assessed using two-tailed Fisher exact probability tests or chi-square contingency tables. For suburb-scale data, Student t-tests were used to compare suburb data (e.g., average income) with adoption fee. The sequential Bonferroni correction was applied to the t-test data to ensure a significance level of 0.05 across the full range of tests [30].

The two mandatory legislative variables (collars and registration) and whether cats roam beyond adopter residences, were all assessed using mixed-effect hierarchal models. The dependent variable in each case was binary (compliant or not compliant) and the predictors were whether the adoption was free or paid, age of the cat in months, cat sex, gender of the adopter, and whether or not they had adopted from a shelter before. We applied a mixed-model approach because 10 pairs of cats were adopted into the same households. Therefore, the household was treated as a random effect. Effects with $p$-values between 0.01 and 0.05 were considered as offering modest significance and effects with $p<0.01$ as offering stronger evidence of a difference. Given the range of times post-adoption when questionnaires were administered, Fisher exact tests were used to assess any effect of number of months cats were owned at time of survey ( $<6$ months, $<12$ months, $<18$ months) against compliance with collar, registration and roaming variables. Sequential Bonferroni corrections were applied because of the multiple tests.

\subsection{Ethics Approval}

This research was carried out in accordance with Murdoch University's Human Ethics Approval 2014-099. 


\section{Results}

\subsection{Profile of Responses}

Of the 100 free cat adopters surveyed, 69 responses were received (32 email; 18 letter; 19 telephone; $69 \%$ reply rate), representing 65 singles, and four pairs of cats (total $n=73$; Table 1 ). Of 120 normal-fee cat adopter surveys, 72 responses were received (68 email; 4 telephone; $60 \%$ reply rate), representing 66 single and 6 pairs of cats (total $n=78$; Table 1). The response rates of the two groups did not differ significantly (two-tailed comparison of proportions, $z=1.39, p=0.16$ ).

Table 1. Demographics of 73 free cats adopted (4 pairs) and 78 normal-fee cats (6 pairs) adopted from the Cat Haven shelter, WA, in 2013-2015.

\begin{tabular}{|c|c|c|}
\hline Cat Demographics & Free Cats $n=73(\%)$ & Normal-Fee Cats $n=78(\%)$ \\
\hline \multicolumn{3}{|l|}{ Cat Ages } \\
\hline Age range & $1-16$ years & $1-14$ years \\
\hline Age mean & 4 years & 3 years \\
\hline \multicolumn{3}{|l|}{ Cat Sex } \\
\hline TOTAL females & $52(71)$ & $57(73)$ \\
\hline Female adults & $46(63)$ & $54(69)$ \\
\hline Female matures & $6(8)$ & $3(4)$ \\
\hline TOTAL males & $21(29)$ & $21(27)$ \\
\hline Male adults & $16(22)$ & $19(24)$ \\
\hline Male matures & $5(7)$ & $2(3)$ \\
\hline \multicolumn{3}{|l|}{ Pelage Varieties } \\
\hline Blacks and Browns & $9(12)$ & $6(8)$ \\
\hline Calicos and Tortoiseshells & $8(11)$ & $17(22)$ \\
\hline Brindles and Gingers & $9(12)$ & $4(5)$ \\
\hline Greys and Whites & $9(12)$ & $13(17)$ \\
\hline Piebalds & $12(16)$ & $13(17)$ \\
\hline Tabbies & $26(36)$ & $25(32)$ \\
\hline \multicolumn{3}{|l|}{ Cat Fate } \\
\hline Alive and Retained by original adopter & $68(93)$ & $75(96)$ \\
\hline${ }^{1}$ Dead & $3(4)$ & $0(0)$ \\
\hline${ }^{2}$ Returned to shelter & $0(0)$ & $3(4)$ \\
\hline${ }^{3}$ Rehomed privately & $1(1)$ & $0(0)$ \\
\hline${ }^{4}$ Ran away & $1(1)$ & $0(0)$ \\
\hline
\end{tabular}

${ }^{1}$ One cat died of cancer, one of liver disease and the third cat was hit by a car two weeks after adoption. ${ }^{2}$ One mother-son pair of cats were returned to Cat Haven because of fighting with other cats in the household. The third cat was maladjusted to indoor-only living. ${ }^{3}$ One cat developed a bond with a relative of the original adopter and was permanently rehomed with the relative. ${ }^{4}$ Indoor-only cat did not return home after escaping through a door accidentally left open.

\subsection{Cat Demographics}

The demographics of cats did not differ between free and normal-fee adoptions (Table 1). Nearly two thirds of the adoptions in each group were female cats, with no significant association between adoption fee and cat sex (two-tailed Fisher exact test $p=0.86$ ), age category of the cats (two-tailed Fisher exact test $p=0.11$ ), or pelage (chi-squared contingency table $\chi_{1,5}=6.39, p=0.27$ ). The fate of cats was also similar between the two groups (Table 1), using the categories of 'Alive and retained by original adopter' and pooled fewer frequencies of 'Dead', 'Ran Away', 'Rehomed' and Returned' (two-tailed Fisher exact test $p=0.48$ ). No returned cat had a history of failed adoptions. Adopters reported near-identical incidences of medical and behavioural problems for free and normal-fee cats (Table 2). 
Table 2. Incidence of medical and behavioural problems in 73 free, and 78 normal-fee cats, as reported by WA adopters surveyed in 2013-2015.

\begin{tabular}{ccc}
\hline Reported Cat Problems & Free Cats $\boldsymbol{n = 7 3 ~ ( \% )}$ & Normal-Fee Cats $\boldsymbol{n}=\mathbf{7 8}(\mathbf{\%})$ \\
\hline No medical or behavioural problems & $56(77)$ & $60(77)$ \\
Medical problems & & \\
No medical problems & $68(93)$ & $73(93.5)$ \\
Skin rash/Systemic infection & $5(7)$ & $4(5)$ \\
Other & $0(0)$ & $1(1.5)$ \\
Number receiving veterinary treatment & $4(80)$ & $4(80)$ \\
Behavioural problems & & \\
No behavioural problems & $61(83.5)$ & $64(82)$ \\
Shy/Scared & $12(16.5)$ & $3(21.5)$ \\
Number with $\geq 1$ behavioural problems & $4(33)$ & $6(43)$ \\
Aggressive towards owners/Destructive & $2(16.5)$ & $5(36)$ \\
Fights with other pets & $4(33)$ & $0(0)$ \\
Toileting issues & $3(25)$ & $2(14)$ \\
Unhappy as inside-only cat & $0(0)$ & $60(77)$ \\
No medical or behavioural problems & $56(77)$ &
\end{tabular}

\subsection{Adopter Profiles}

The gender profiles of free and normal-fee cat adopters were very similar (Table 3), with more female than male adopters in both samples (two-tailed Fisher exact test $p=0.82$ ). More free- than normal-fee cat adopters were adopting from a shelter for the first time (Table 3; two-tailed Fisher exact test $p=0.04$ ). There was also a difference in the way in which adopters learned about the existence of the Cat Haven shelter (Table 3; word-of-mouth, media and pooled miscellaneous categories, two-tailed Fisher exact test $p<0.001$ ), with most free cat adopters learning of the Cat Haven through promotion of the adoption-drive using various media (e.g., radio, social media). Normal-fee cat adopters mainly learned about the Cat Haven through word-of-mouth. After sequential Bonferroni correction, Student $\mathrm{t}$-tests revealed no significant differences between adoption fee and any characteristics of suburbs where owners resided (Table 4).

Table 3. Survey responses of free and normal-fee cat adopters including gender, previous adoption history with cat shelters, and how adopters originally learned about the Cat Haven shelter, WA. Over the free adoption-drive weekend, four survey respondents adopted a pair of cats; and six pairs of normal-fee cats were adopted during the normal non-promotional period.

\begin{tabular}{cccc}
\hline Variable & Responses & Free Adopters \\
$\mathbf{n = 6 9 ( \% )}$ & $\begin{array}{c}\text { Normal-Fee Adopters } \\
\mathbf{n = 7 2} \mathbf{( \% )}\end{array}$ \\
\hline \multirow{2}{*}{ Owner gender } & Female & $59(86)$ & $60(83)$ \\
& Male & $10(14)$ & $12(17)$ \\
\hline \multirow{3}{*}{ Adoption history } & Never adopted from a shelter & $57(83)$ & $46(64)$ \\
& Previously adopted from a shelter & $10(14)$ & $20(28)$ \\
& Unknown & $2(3)$ & $6(8)$ \\
Information source & Media & $40(58)$ & $12(17)$ \\
& Word-of-mouth & $21(30)$ & $38(53)$ \\
& Miscellaneous & $2(3)$ & $9(12)$ \\
& Unknown & $6(9)$ & $13(18)$ \\
\hline
\end{tabular}


Table 4. Student $t$-test results for difference between 12 Western Australian suburb statistics [23] and cat adoption fee. No result is significant after sequential Bonferroni correction.

\begin{tabular}{cccc}
\hline Suburb Parameters & $\boldsymbol{t}$-Test Value & df & $p$-Value \\
\hline Distance from shelter $(\mathrm{km})$ & 1.41 & 129 & 0.16 \\
Number of people $/ \mathrm{km}^{2}$ & -2.29 & 148 & 0.04 \\
Average number of people/household & 1.00 & 144 & 0.32 \\
Median age of people in suburb & -0.65 & 138 & 0.52 \\
People with language other than English $(\%)$ & 0.25 & 142 & 0.80 \\
Average personal salary & -0.09 & 135 & 0.93 \\
Total suburb income (millions \$) & -0.49 & 149 & 0.63 \\
Population with post-school qualification $(\%>15$ years age) & -1.08 & 143 & 0.28 \\
Unemployment rate & 0.46 & 146 & 0.64 \\
Working population (\% 15-64 years age) & -0.69 & 126 & 0.49 \\
Number of births & 0.65 & 146 & 0.51 \\
Number of deaths & -0.35 & 149 & 0.72 \\
\hline
\end{tabular}

\subsection{Compliance with Regulations}

Survey responses were tallied to assess compliance with three responsible cat ownership variables under state legislation (two mandatory, one implied; Table 5).

Table 5. Compliance of people adopting free and normal-fee cats with three variables of responsible cat ownership under WA legislation [25]. Wearing collars and registering cats with local councils are mandatory. Restricting cats to private property is recommended.

\begin{tabular}{cccc}
\hline Variable & Responses & Free Cats $\boldsymbol{n = 7 3 ( \% )}$ & Normal-Fee Cats $\boldsymbol{n}=\mathbf{7 8}(\mathbf{\%})$ \\
\hline \multirow{3}{*}{ Collar } & Wears collar & $50(69)$ & $51(65)$ \\
& Does not wear collar & $22(30)$ & $22(28)$ \\
& Unknown & $1(1)$ & $5(6)$ \\
\hline \multirow{3}{*}{ Registration } & Registered with council & $55(75)$ & $63(81)$ \\
& Not registered with council & $17(23)$ & $10(13)$ \\
& Unknown & $1(1)$ & $5(6)$ \\
\hline \multirow{3}{*}{ Roaming } & Allowed to roam freely & $39(53)$ & $43(55)$ \\
& Not allowed to roam freely & $34(47)$ & $34(44)$ \\
& Unknown & $0(0)$ & $1(1)$ \\
\hline
\end{tabular}

Two-tailed Fisher exact tests revealed there was no difference between free and normal-fee adopters' adherence to collar $(p>0.99)$, registration $(p=0.14)$ or roaming $(p=0.87)$ legislation. After sequential Bonferroni correction, the number of months cats had been owned when surveyed did not affect whether cats wore collars (free $p>0.99$, normal-fee $p=0.10$ ) or were registered (free $p>0.99$, normal-fee $p=0.24)$. Months owned at survey also did not affect whether free cats $(p=0.24)$, or normal-fee cats $(p=0.02)$ could roam freely. Mixed-effect hierarchal models detected only one modest association between cat predictors and husbandry response variables, which was a greater likelihood for male adopters to allow cats to roam (Table 6). 
Table 6. Mixed-effect hierarchal modelling used to assess relationship between free and normal-fee adopter husbandry response variables and adopted cat predictors. Effects with $p$-values between 0.01 and 0.05 were considered as offering modest significance and effects with $p<0.01$ as offering stronger evidence of a difference.

\begin{tabular}{|c|c|c|c|c|}
\hline $\begin{array}{l}\text { Husbandry } \\
\text { Variables }\end{array}$ & Cat Predictors & $\begin{array}{l}\text { Estimate } \pm \\
\text { Std Error }\end{array}$ & $p$-Value & $\begin{array}{c}\text { Interpretation of Husbandry } \\
\text { vs. Adoption Fee }\end{array}$ \\
\hline $\begin{array}{l}\text { Cat wears } \\
\text { collar }\end{array}$ & $\begin{array}{c}\text { Free vs. normal fee } \\
\text { Owner gender } \\
\text { Previous adoption history } \\
\text { Cat age in months } \\
\text { Cat sex }\end{array}$ & $\begin{array}{c}-0.006 \pm 0.41 \\
0.20 \pm 0.56 \\
0.47 \pm 0.51 \\
-0.20 \pm 0.19 \\
0.67 \pm 0.49\end{array}$ & $\begin{array}{l}0.99 \\
0.72 \\
0.35 \\
0.30 \\
0.17\end{array}$ & $\begin{array}{l}\text { Likelihood of owner placing } \\
\text { collar on cat does not vary } \\
\text { with adoption fee }\end{array}$ \\
\hline $\begin{array}{l}\text { Cat is } \\
\text { registered }\end{array}$ & $\begin{array}{c}\text { Free vs. normal fee } \\
\text { Owner gender } \\
\text { Previous adoption history } \\
\text { Cat age in months } \\
\text { Cat sex }\end{array}$ & $\begin{array}{c}0.71 \pm 0.49 \\
0.38 \pm 0.69 \\
-0.58 \pm 0.54 \\
-0.38 \pm 0.20 \\
0.91 \pm 0.61\end{array}$ & $\begin{array}{l}0.15 \\
0.58 \\
0.28 \\
0.06 \\
0.13\end{array}$ & $\begin{array}{l}\text { Likelihood of owner } \\
\text { registering cat with local } \\
\text { council does not vary with } \\
\text { adoption fee }\end{array}$ \\
\hline $\begin{array}{l}\text { Cat is } \\
\text { allowed to } \\
\text { roam }\end{array}$ & $\begin{array}{c}\text { Free vs. normal fee } \\
\text { Owner gender } \\
\text { Previous adoption history } \\
\text { Cat age in months } \\
\text { Cat sex }\end{array}$ & $\begin{array}{l}-0.26 \pm 0.46 \\
-1.33 \pm 0.67 \\
-0.03 \pm 0.54 \\
-0.03 \pm 0.22 \\
-0.15 \pm 0.48\end{array}$ & $\begin{array}{l}0.57 \\
0.05 \\
0.95 \\
0.87 \\
0.75\end{array}$ & $\begin{array}{l}\text { Likelihood of owner } \\
\text { restricting cat to residence } \\
\text { property does not vary with } \\
\text { adoption fee }\end{array}$ \\
\hline
\end{tabular}

\subsection{Shelter Perception of the Free Adult Cat Adoption-Drive}

Public response to the free adult cat adoption-drive was so high that all shelter-housed cats were adopted before close of the first day, and over the course of the weekend healthy adults in foster-care were recalled to the shelter to fill newly created vacancies. In total, 137 healthy adult and mature cats were adopted during the free weekend and thereby spared euthanasia. During the week including the free weekend in 2015, 182 adult and mature cats were adopted. In comparison with the mean adoptions of adult and mature cats for all non-promotional weeks in 2015 (34.11 cats), the free adoption-drive increased weekly total cat adoptions by approximately $533 \%$ (Figure 1). Adoptions from the same week in 2014 totalled 22 cats, considerably less than the 182 in 2015.

The free adult adoption-drive also substantially increased adoptions of full-fee kittens, with 154 kittens adopted during the free weekend. During the week including the free weekend in 2015, 199 kittens were adopted. In comparison with the mean adoptions of kittens for all non-promotional weeks in 2015 (52.25 kittens), the free adoption-drive increased weekly total kitten adoptions by approximately $381 \%$ (Figure 1). Adoptions from the same week in 2014 totalled 73 kittens, considerably less than the 199 in 2015. Adoption of 154 normal-fee kittens during the free weekend created space for new incoming cats and, despite waiving adult and mature cat adoption fees, the Cat Haven CEO R. Robinson states that "More revenue was generated in three days than is normally made in three weeks from purchases of food, accessories and full-fee kittens."

During 2014 and 2015 weekly rates of euthanasia (Figure 2) included no healthy adult cats, in accordance with Cat Haven's long-term goal of zero euthanasia of healthy animals. The main reasons for euthanasia were health (e.g., feline calici virus) or behaviour-related (e.g., feral). Cat Haven CEO R. Robinson commented: "Faced with so many cats (in 2015) we couldn't rehome due to kittens being available, we had to take radical steps. Being an open admission shelter, we simply couldn't continue to keep taking cats in, without getting them out. We had two choices, either give the cats away for free, or euthanize them, Euthanasia was not an option as we felt it was unethical to put healthy and friendly cats to sleep." 


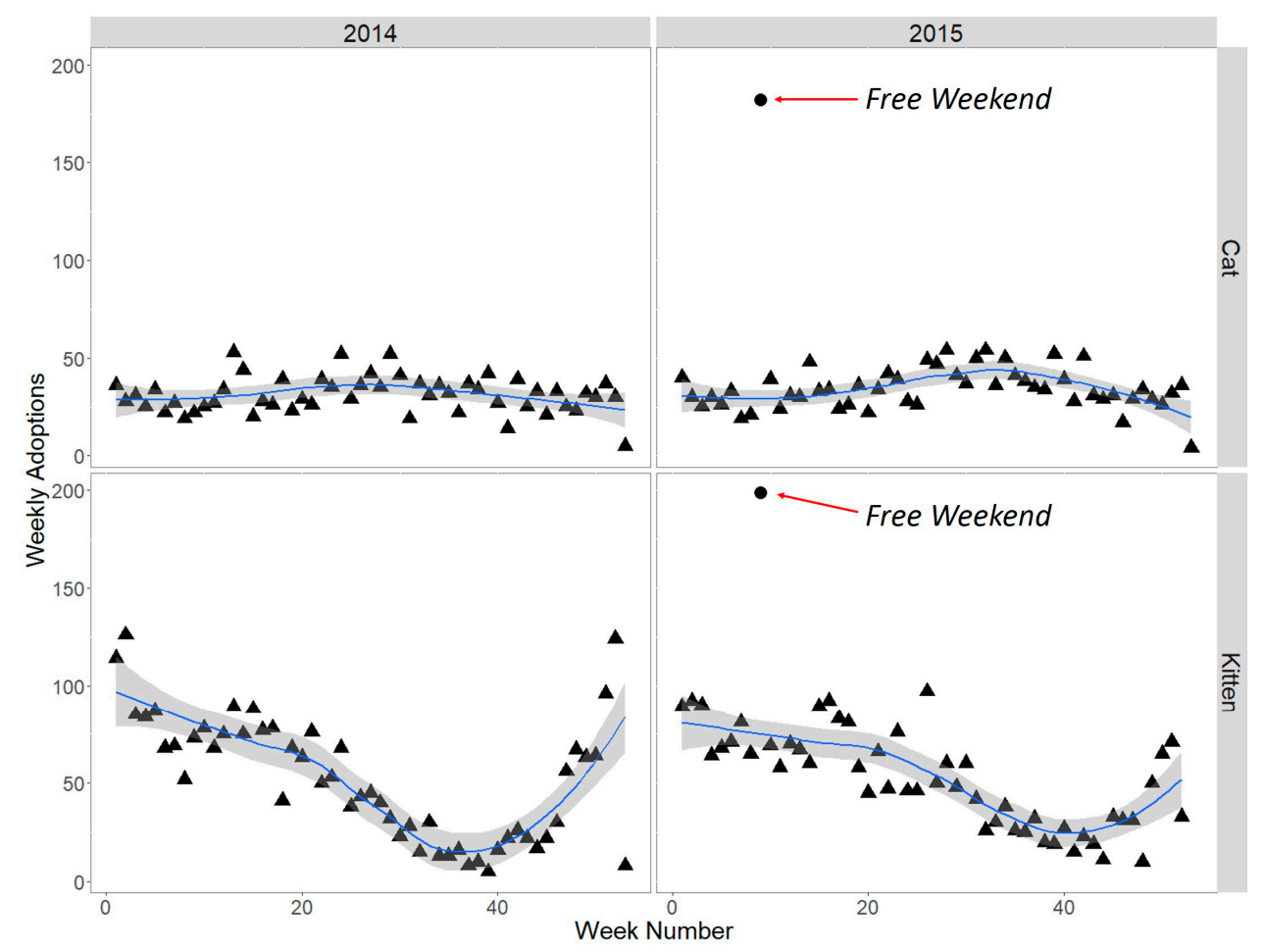

Figure 1. Weekly cat and kitten adoptions 2014-2015 at the Cat Haven, WA, with a loess smoother and $95 \%$ confidence envelope. The free weekend dot indicates the number of adult and mature cats or kittens adopted during the entire week that included the adoption-drive. Free weekends increased cat adoptions by $533 \%$ and kitten adoptions by $381 \%$ relative to 2015 weekly averages.

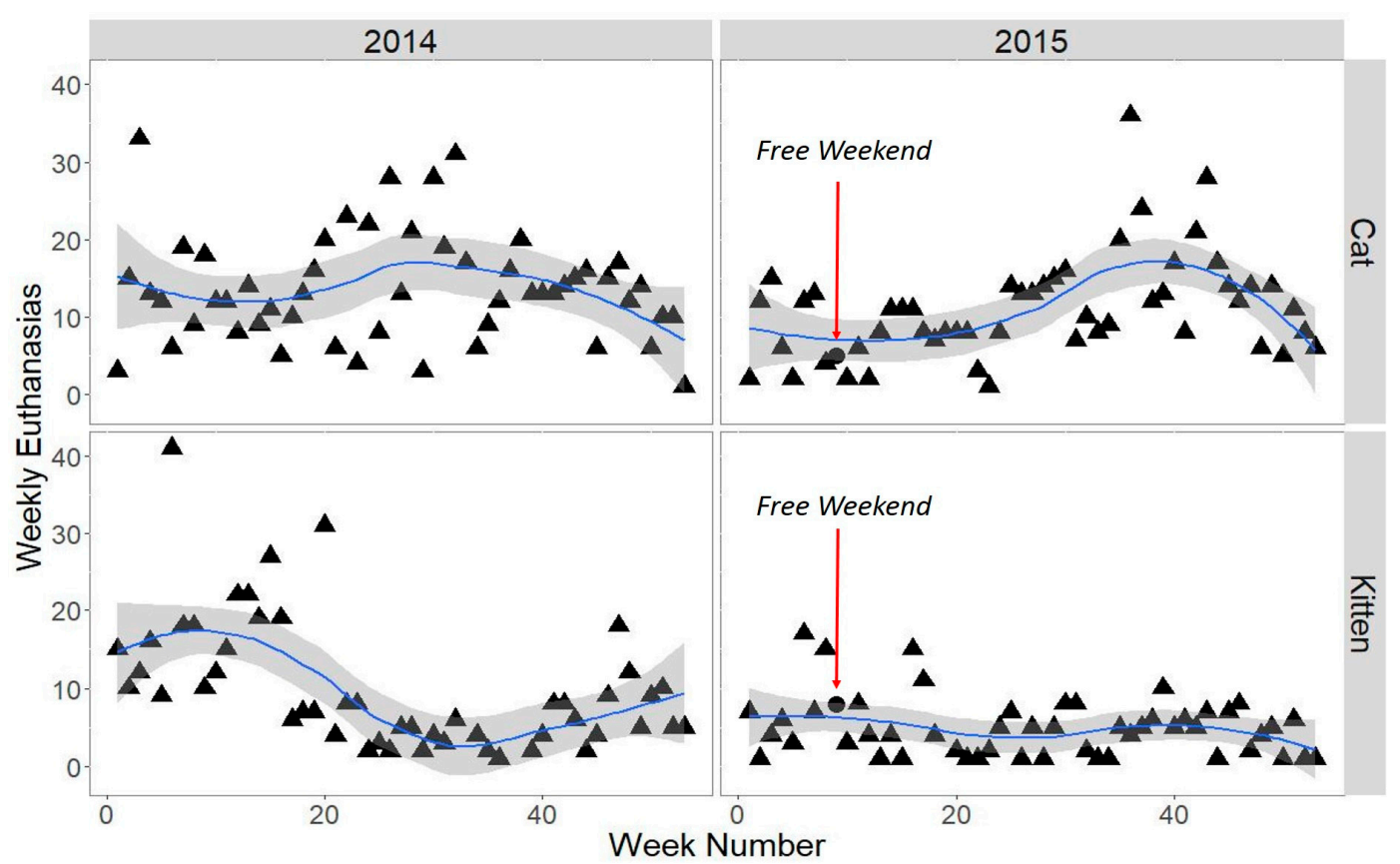

Figure 2. Weekly euthanasia rates for cats and kittens 2014-2015 at the Cat Haven, WA, with a loess smoother and 95\% confidence envelope. The free weekend dot indicates the number of adult and mature cats or kittens euthanised during the entire week that included the adoption-drive. 


\section{Discussion}

In keeping with our predictions, demographics of cats adopted did not differ between free and normal-fee adoptions. Both groups adopted cats of similar age, gender and pelage. Comparison of the fate of cats adopted for free or for a fee, revealed that the majority of cats were alive and retained by their original adopters ( $93 \%$ for free cats, $96 \%$ for normal-fee cats). These findings closely parallel those of an Australian study (state of Queensland [22]) which found that 6-12 months after adoption $91 \%$ of $\$ 20$ cats $(n=126)$ and $90 \%$ of $\geq \$ 99(n=17)$ cats were alive and retained by the original adopter.

Post-adoption, the incidences of medical and behavioural problems were also nearly identical, leaving $77 \%$ of both free- and normal-fee cats alive and well. Treatable medical problems developed in only $7 \%$ of free, and $6.5 \%$ of normal-fee cats (e.g., diarrhoea, skin rash, haematuria), with $80 \%$ of unwell cats receiving veterinary treatment, regardless of adoption fee. Including the three cats returned to the Cat Haven, only $16.5 \%$ of free, and $18 \%$ of normal-fee cats were reported as having any enduring behavioural problems, with free cats mainly shy or hiding in fear (33\%), and normal-fee cats mainly aggressive towards owners or destructive (43\%). Problem behaviours can be common in cats [31,32], and cats are frequently surrendered or returned to shelters if they do not rapidly adjust to their new home, or display antisocial, destructive or inappropriate elimination behaviours [33-36]. For example, over the course of one year, $33 \%$ of cat owners cited behaviour problems as reasons for surrendering their cats to 12 animal shelters in the USA $(n=2168$ [36]). The near-identical low incidence of reported behavioural problems in free and normal-fee cats is consistent with owners in the two groups perceiving the behaviour of their cats similarly, some of which may be natural behaviour that needs appropriate redirection [37].

Overall, there is no evidence that adoption fee influences the selection of cats or their fate with their new owners. Perception of behavioural problems, which is an acknowledged risk for return of adopted cats to a shelter, was also similar between the two groups and likely contributed to the similar fates of cats in both groups.

\subsection{Do Free Adoption-Drives Attract a Different Group of Adopters?}

The care that pet cats receive from owners may be influenced by owner features such as age, income, gender, education, previous experience owning pets, presence of children, and number of emotional bonds with friends [38,39]. However, we found that both free and normal-fee adopters were similar across 12 different demographic variables constructed from ABS suburb-scale data. Where the two groups did differ was in their experience adopting from shelters, with more first-time adopters visiting the Cat Haven during the free adult adoption-drive. It is perhaps unsurprising then that the groups also differed in how they initially learned of the Cat Haven's existence, with free adopters learning about the shelter from media promotions of the free adoption-drive, and normal-fee adopters learning through word-of-mouth. Given that pet cats have extended life expectancies ( 15 years for indoor cats and 10 years for outdoor cats [40]), and that this may limit the number of experienced cat owners who are available to adopt, attracting first-time adopters must be a priority for shelters wishing to decrease euthanasia of healthy cats.

\subsection{Does Adoption Fee Influence Legislative Compliance?}

Adopters of free and normal-fee cats did not significantly differ in their legislative compliance. Both groups were as likely to place collars on cats (69\% free vs. $65 \%$ normal-fee), register them with local municipal councils ( $75 \%$ vs. $81 \%$ ), and allow cats to roam outside and beyond their residence boundary (53\% vs. $55 \%$ ). The high compliance with registration may reflect Cat Haven's legal responsibility to notify local councils with details of adoptions, as well as the shelter's efforts to communicate the responsibilities of all cat owners. Registration is not free (fees vary between municipalities), so cost might be a factor in determining whether or not owners register their animals. 
However, there was no statistically significant difference in the proportion of cats registered between free and normal-fee adopters.

All Australian states and the Australian Capital Territory have legislation governing aspects of cat ownership, but there is little data on compliance and much of that suggests that compliance is variable and low. For example, in 2001 approximately 500,000 dogs and cats were microchipped in New South Wales under legislative requirements, but only 200,000 animals were registered [41]. In one Victorian municipality only $15 \%$ of households registered a cat, substantially below the national cat ownership estimate of $26 \%$ of households [42]. In relation to confinement of pet cats, many owners appear to make a decision about husbandry based on what they believe is best for their cat's welfare, and do not always comply with legislation that they feel compromises welfare [43]. Welfare considerations may also apply when owners choose whether or not fit a collar, because of perceptions that collars are hazardous to cats despite data showing that the risk is low and the benefits of simple identification high [44,45]. Overall, decisions about compliance are complex and the modest compliance noted in our samples may or may not be representative of national trends.

\subsection{Why Are Free Adoptions Good for Cats?}

Preventing euthanasia of healthy adult cats is an obvious, immediate positive outcome of reducing crowding by offering occasional free adult cat adoption-drives $[15,46]$. Moving adult and mature cats through shelter systems quickly also benefits general welfare by minimising exposure to diseases such as upper respiratory tract infections or ringworm [47-51], and to unpredictable, sustained or novel stimuli such as handing by visitors [52], that can cause stress-related urinary tract infections [53], and behavioural issues such as abnormal eating or grooming [54]; elimination [55]; and hypervigilance [53]. Furthermore, because many factors influence the likelihood of adoption including cat sex (e.g., males preferred [15]), appearance (e.g., rare breeds preferred, prejudices against darker coat patterns $[18,56,57])$, and perceptions of cat personality (e.g., playfulness and willingness to interact with people increases adoptions [58-60]); in a free adoption scenario where cats are being rapidly rehomed, the less popular animals may have a better chance of selection.

Pet cats surrendered to shelters have higher stress scores than stray cats, and this can negatively affect their health, length of stay and increase chances of euthanasia [61]. If cats enter an adoption-surrender cycle they may become traumatised and deemed unsuitable for rehoming. If free cat adoption-drives are used in times of crowding (or in anticipation of them) and are accompanied by rigorous pre-adoption counselling (as practised by the Cat Haven in this study) and supported by post-adoption advice or follow-up, then there is no reason to predict that free adult cats will be surrendered more readily than those sold for a fee.

\subsection{Why Are Free Adoptions Good for Shelters?}

Rehoming adult cats will reduce euthanasia rates and the associated risk of staff suffering 'trauma fatigue' $[19,20]$. Foster carers, who are often a pivotal resource relied on by animal shelters to help deal with burgeoning numbers of unwanted and juvenile animals [62,63], are also relieved when all surrendered animals can be accommodated within the shelter. Overall, shelters offering free adoptions will save the cost of long-term housing and provision of environmental enrichment for animals with lower adoption potential. Resources may then be redirected towards costs of caring for kittens or public education programs that may reduce abandonment of cats (e.g., increasing community desexing rates of cats). Furthermore, Cat Haven CEO R. Robinson noted "the weekend was a remarkable success. Adopters made generous donations, and bought quality food, and accessories for the cats they adopted."

Free adoption-drives afford shelters with further opportunities to educate new, and experienced adopters, prior to point-of-sale. This education in the form of pre-adoption counselling, is crucial to breaking adoption-return cycles and reducing euthanasia. While many shelters routinely provide take-home leaflet information about cat behaviour, disease and responsible ownership, shelters are 
often unable to counsel potential adopters about their motivations for adopting, and what pet would be most suitable for their lifestyle. Yet, adoptions frequently fail as a result of unrealistic owner expectations about the role a cat will play in the family and of its temperament [64-66]. According to Podberscek [67] (p. 368), "Sometimes simple education about why an animal behaves in a certain way is enough to make the animal more acceptable in the eyes of the owner." Therefore, offering pre-adoption counselling at all times may benefit shelters, cats and adopters in a number of ways. Firstly, new and experienced adopters will benefit equally from being matched with the best possible cat candidate for adoption, which should translate into faster acclimation to new homes, less stress for owners and fewer returned animals. Secondly, whether or not an adoption fee is paid, pre-adoption counselling for both new and experienced adopters will provide shelter staff with opportunity to assess the expectations of people adopting cats.

\subsection{Limitations of the Study}

This paper reports a single case study, which may not be representative of other shelters in other jurisdictions. However, it has value in adding data to an important question where there are few other studies, which also share the limitation of being geographically restricted [21,22]. Furthermore, this study adds the unique element of assessing legislative compliance to complement existing work on attachment levels and caring practices of owners [21], or outcomes for adopted cats [22]. While sample sizes for cats adopted were modest in our study (151), this is also true of existing studies (173 cats in [21], and up to 248 adult cats in [22]. The value comes from multiple studies contributing to different aspects of the outcomes of free adoptions, which all reach a similar conclusion that there were no serious negative outcomes for cats or participating shelters.

\section{Conclusions}

The Cat Haven, in common with many shelters globally, seeks to rehome unwanted cats and minimise or eliminate euthanasia of healthy cats. Owners who adopted free cats did not differ from fee paying adopters in demographics or compliance with husbandry legislation. Free cat adoption-drives are likely to attract first-time cat adopters, especially if shelters use a variety of media. This will help deal with issues of saturation among cat owners. Animal shelters should not be dissuaded from free adoption promotions during times of crowding, and may even wish to hold one in anticipation of a high demand for housing. Although these conclusions apply to one local shelter, they are also consistent with the limited literature on the topic of free and discounted adoptions [21,22].

Acknowledgments: This research was carried out in accordance with Murdoch University's Human Ethics Approval 2014-099. Thank you to the Cat Haven CEO, staff and volunteers, and to the cat adopters who participated in follow-up surveys. We also thank the Australian Pet Welfare Foundation for partially funding this research. Processing costs for this paper were covered by the Special Issue sponsors, Maddie's Fund ${ }^{\circledR}$, Found Animals, and The Humane Society of the United States (HSUS).

Author Contributions: Heather M. Crawford collected the data, ran most analyses and led the writing of the manuscript. Joseph B. Fontaine wrote R code for statistical analyses and contributed to writing the manuscript. Michael C. Calver assisted in study design and writing of the manuscript.

Conflicts of Interest: The authors declare no conflict of interest. 


\section{Appendix A}

Table A1. Post-adoption surveys using email, letter and telephone questionnaires were identical for people who adopted cats for free, or for a normal-fee.

\begin{tabular}{cc}
\hline $\begin{array}{c}\text { (1) OWNER DETAILS } \\
\text { Name and Surname }\end{array}$ & (2) CAT DETAILS \\
\hline Gender & Number of cats adopted \\
\hline Address & Cat name \\
\hline Contact details & Cat sex \\
\hline (3) SHELTER EXPERIENCE & Approximate age when adopted \\
\hline $\begin{array}{c}\text { How did you come to learn of Cat Haven? } \\
\text { how many years ago? }\end{array}$ & (4) CAT HUSBANDRY your cat wear a collar? Why/why not? \\
\hline $\begin{array}{c}\text { Was the information provided at time of adoption } \\
\text { and the take-home-info-pack of use to you? If yes } \\
\text { how? }\end{array}$ & $\begin{array}{c}\text { Is your cat given access to the outdoors? Why/why } \\
\text { not? }\end{array}$ \\
$\begin{array}{c}\text { What price did you pay for the cat? } \\
\text { In your view can the Cat Haven improve anything? }\end{array}$ & $\begin{array}{c}\text { If yes at what time and how often does your cat have } \\
\text { access to the outdoors? }\end{array}$ \\
\hline
\end{tabular}

\section{References}

1. Pet Data Report 2017. Pet Food Manufacturer's Association: London, UK. Available online: www.pfma.org. uk (accessed on 8 May 2017).

2. Facts \& Figures 2014. European Pet Food Industry Federation: Brussels, Belgium. Available online: www. fediaf.org (accessed on 10 May 2016).

3. National Pet Owners Survey 2015-2016. Available online: www.americanpetproducts.org (accessed on 1 February 2016).

4. Companion Animals in New Zealand 2016. New Zealand Companion Animal Council Inc.: Auckland, New Zealand. Available online: www.nzcac.org.nz (accessed on 2 February 2016).

5. Pet Ownership in Australia 2016. Animal Medicines Australia: New South Wales, Australia. Available online: www.animalmedicinesaustralia.org.au (accessed on 10 October 2016).

6. Faya, M.; Carranza, A.; Priotto, M.; Abeya, M.; Diaz, J.D.; Gobello, C. Domestic queens under natural temperate photoperiod do not manifest seasonal anestrus. Anim. Reprod. Sci. 2011, 129, 78-81. [CrossRef] [PubMed]

7. Jennett, A.L.; Jennett, N.M.; Hopping, J.; Yates, D. Evidence for seasonal reproduction in UK domestic cats. J. Feline Med. Surg. 2016, 18, 804-808. [CrossRef] [PubMed]

8. Marston, L.C.; Bennett, P.C. Admissions of cats to animal welfare shelters in Melbourne, Australia. J. Appl. Anim. Welf. Sci. 2009, 12, 189-213. [CrossRef] [PubMed]

9. Nutter, F.B.; Levine, J.F.; Stoskopf, M.K. Reproductive capacity of free-roaming domestic cats and kitten survival rate. J. Am. Vet. Med. Assoc. 2004, 225, 1399-1402. [CrossRef] [PubMed]

10. Ortega-Pacheco, A.; Concha-Guillermo, H.; Segura-Correa, J.; Jimenez-Coello, M. Seasonal reproductive activity of domestic queens (Felis catus) in the Tropics of Mexico. Reprod. Domest. Anim. 2012, 47, 52-54. [CrossRef] [PubMed]

11. Rinzin, K.; Stevenson, M.A.; Probert, D.W.; Bird, R.G.; Jackson, R.; French, N.P.; Weir, J.A. Free-roaming and surrendered dogs and cats submitted to a humane shelter in Wellington, New Zealand, 1999-2006. N. Z. Vet. J. 2008, 56, 297-303. [CrossRef] [PubMed]

12. Root, M.V.; Johnston, S.D.; Olson, P.N. Estrous length, pregnancy rate, gestation and parturition lengths, litter size, and juvenile mortality in the domestic cat. J. Am. Anim. Hosp. Assoc. 1995, 31, 429-433. [CrossRef] [PubMed] 
13. Australia National Statistics 2015-2016. Royal Society for the Prevention of Cruelty to Animals. Available online: www.rspca.org.au (accessed on 2 February 2017).

14. Maubach, B. Benchmarking Best Practices in Decreasing Animal Shelter Euthanasia Rates Across the West Coast. Honours in Business Management. Bachelor's Thesis, University of Arizona, Tucson, AZ, USA, 2014.

15. Alberthsen, C.; Rand, J.S.; Bennett, P.C.; Paterson, M.; Lawrie, M.; Morton, J.M. Cat admissions to RSPCA shelters in Queensland, Australia: Description of cats and risk factors for euthanasia after entry. Aust. Vet. J. 2013, 91, 35-42. [CrossRef] [PubMed]

16. Večerek, V.; Kubesová, K.; Voslářová, E.; Bedáňová, I. Rates of death and euthanasia for cats in no-kill shelters in the Czech Republic. Acta Vet. Brno 2017, 86, 109-115. [CrossRef]

17. Ardila Galvis, J.O.; Santos Baquero, O.; Dias, R.A.; Ferreira, F.; Nestori Chiozzotto, E.; Grisi-Filho, J.H.H. Monitoring techniques in the capture and adoption of dogs and cats. Geospat. Health 2015, 10, 158-162. [CrossRef] [PubMed]

18. Brown, W.P.; Morgan, K.T. Age, breed designation, coat color, and coat pattern influenced the length of stay of cats at a no-kill shelter. J. Appl. Anim. Welf. Sci. 2015, 18, 169-180. [CrossRef] [PubMed]

19. Reeve, C.L.; Rogelberg, S.G.; Spitzmüller, C.; DiGiacomo, N. The caring-killing paradox: Euthanasia-related strain among animal-shelter workers. J. Appl. Soc. Psychol. 2005, 35, 119-143. [CrossRef]

20. Rohlf, V.; Bennett, P. Perpetration-induced traumatic stress in persons who euthanize nonhuman animals in surgeries, animal shelters, and laboratories. Soc. Anim. 2005, 13, 201-219. [CrossRef] [PubMed]

21. Weiss, E.; Gramann, S. A comparison of attachment levels of adopters of cats: Fee-based adoptions versus free adoptions. J. Appl. Anim. Welf. Sci. 2009, 12, 360-370. [CrossRef] [PubMed]

22. Zito, S.; Paterson, M.; Vankan, D.; Morton, J.; Bennett, P.; Phillips, C. Determinants of cat choice and outcomes for adult cats and kittens adopted from an Australian animal shelter. Animals 2015, 5, 276-314. [CrossRef] [PubMed]

23. Australian Bureau of Statistics 2011-2013. Available online: www.abs.gov.au (accessed on 15 July 2015).

24. Getting 2 Zero: Australian Cat Action Plan 2014-2016. Available online: www.g2z.org.au (accessed on 15 December 2016).

25. Government of Western Australia. Cat Act 2011; Government of Western Australia: Perth, Australia, 2011. Available online: www.dlgc.wa.gov.au/AdviceSupport/Pages/Cats-and-dogs.aspx (accessed on 5 April 2016).

26. Wickham, H. ggplot2: Elegant Graphics for Data Analysis, 2nd ed.; Springer Nature: New York, NY, USA, 2016; ISBN 978-0038079814103.

27. R Core Team. R: A Language and Environment for Statistical Computing; R Foundation for Statistical Computing: Vienna, Austria, 2014.

28. VassarStats: Website for Statistical Computation 1998-2017. Available online: www.vassarstats.net (accessed on 29 October 2017).

29. Bates, D.; Mäechler, M.; Bolker, B.; Walker, S. Fitting linear mixed-effects models using lme4. J. Stat. Softw. 2015, 67, 1-48. [CrossRef]

30. Holm, S. A simple sequentially rejective multiple test procedure. Scand. J. Stat. 1979, 6, 65-70.

31. Heidenberger, E. Housing conditions and behavioural problems of indoor cats as assessed by their owners. Appl. Anim. Behav. Sci. 1997, 52, 345-364. [CrossRef]

32. Seksel, K. Chapter 13-Behavior Problems. In The Cat: Clinical Medicine and Management, 1st ed.; Little, S.E., Ed.; W.B. Saunders: Saint Louis, MO, USA, 2012; pp. 211-225.

33. Casey, R.A.; Vandenbussche, S.; Bradshaw, J.W.S.; Roberts, M.A. Reasons for relinquishment and return of domestic cats (Felis silvestris catus) to rescue shelters in the UK. Anthrozoös 2009, 22, 347-358. [CrossRef]

34. Miller, D.D.; Staats, S.R.; Partlo, C.; Rada, K. Factors associated with the decision to surrender a pet to an animal shelter. J. Am. Vet. Med. Assoc. 1996, 209, 738-742. [PubMed]

35. New, J.C., Jr.; Salman, M.D.; King, M.; Scarlett, J.M.; Kass, P.H.; Hutchison, J.M. Characteristics of shelter-relinquished animals and their owners compared with animals and their owners in U.S. pet-owning households. J. Appl. Anim. Welf. Sci. 2000, 3, 179-201. [CrossRef]

36. Salman, M.D.; New, J.J.G.; Scarlett, J.M.; Kass, P.H.; Ruch-Gallie, R.; Hetts, S. Human and animal factors related to relinquishment of dogs and cats in 12 selected animal shelters in the United States. J. Appl. Anim. Welf. Sci. 1998, 1, 207-226. [CrossRef] [PubMed]

37. Jongman, E.C. Adaptation of domestic cats to confinement. J. Vet. Behav. 2007, 2, 193-196. [CrossRef] 
38. Adamelli, S.; Marinelli, L.; Normando, S.; Bono, G. Owner and cat features influence the quality of life of the cat. J. Appl. Anim. Behav. Sci. 2005, 94, 89-98. [CrossRef]

39. Finkler, H.; Terkel, J. The contribution of cat owners' attitudes and behaviours to the free-roaming cat overpopulation in Tel Aviv, Israel. Prev. Vet. Med. 2012, 104, 125-135. [CrossRef] [PubMed]

40. Brunner, D.; Stall, S. The Cat Owner's Manual: Operating Instructions, Troubleshooting Tips, and Advice on Lifetime Maintenance, 1st ed.; Quirk Books: Philadelphia, PA, USA, 2004.

41. Pert, T.A. New South Wales Companion Animals Act 1998. Funding_-Is it adequate for animal management? In Proceedings of the 10th National Conference on Urban Animal Management in Australia, Melbourne, Australia, 29-31 August 2001; pp. 49-52.

42. Scheele, M. Cat pounds-Manningham City Council perspective. In Proceedings of the 10th National Conference on Urban Animal Management in Australia, Brisbane, Australia, 29-31 August 2001; pp. 69-71.

43. Van de Kuyt, N. Turning Research into Reality: How Councils can Use Findings from a Survey to Help Manage Pets in the Community. Available online: https://www.google.com. hk/url?sa=t\&rct=j\&q=\&esrc=s\&source=web\&cd=1\&cad=rja\&uact=8\&ved=0ahUKEwjWwY_I-e_ XAhVFlpQKHQDtDzsQFggqMAA\&url=https\%3A\%2F\%2Fwww.parliament.vic.gov.au\%2Fimages\% 2Fstories\%2Fcommittees\%2FSCEI\%2FDogs_Inquiry\%2FSubs\%2FSubmission_177e_-_Diana_Rayment. pdf\&usg=AOvVaw1LaZCJH8gmm9byBOvkgskX (accessed on 10 October 2017).

44. Calver, M.C.; Adams, G.; Clark, W.; Pollock, K.H. Assessing the safety of collars used to attach predation deterrent devices and ID tags to pet cats. Anim. Welf. 2013, 22, 95-105. [CrossRef]

45. Weiss, E.; Slater, M.R.; Lord, L.K. Retention of provided identification for dogs and cats seen in veterinary clinics and adopted from shelters in Oklahoma City, OK, USA. Prev. Vet. Med. 2011, 101, 265-269. [CrossRef] [PubMed]

46. Morris, K.N.; Wolf, J.L.; Gies, D.L. Trends in intake and outcome data for animal shelters in Colorado, 2000 to 2007. J. Am. Vet. Med. Assoc. 2011, 238, 329-336. [CrossRef] [PubMed]

47. Bannasch, M.J.; Foley, J.E. Epidemiologic evaluation of multiple respiratory pathogens in cats in animal shelters. J. Feline Med. Surg. 2005, 7, 109-119. [CrossRef] [PubMed]

48. Dinnage, J.D.; Scarlett, J.M.; Richards, J.R. Descriptive epidemiology of feline upper respiratory tract disease in an animal shelter. J. Feline Med. Surg. 2009, 11, 816-825. [CrossRef] [PubMed]

49. McManus, C.M.; Levy, J.K.; Andersen, L.A.; McGorray, S.P.; Leutenegger, C.M.; Gray, L.K.; Hilligas, J.; Tucker, S.J. Prevalence of upper respiratory pathogens in four management models for unowned cats in the Southeast United States. Vet. J. 2014, 201, 196-201. [CrossRef] [PubMed]

50. Steneroden, K.K.; Hill, A.E.; Salman, M.D. A needs-assessment and demographic survey of infection-control and disease awareness in western US animal shelters. Prev. Vet. Med. 2011, 98, 52-57. [CrossRef] [PubMed]

51. Tanaka, A.; Wagner, D.C.; Kass, P.H.; Hurley, K.F. Associations among weight loss, stress, and upper respiratory tract infection in shelter cats. J. Am. Vet. Med. Assoc. 2012, 240, 570-576. [CrossRef] [PubMed]

52. Gourkow, N.; Fraser, D. The effect of housing and handling practices on the welfare, behaviour and selection of domestic cats (Felis sylvestris catus) by adopters in an animal shelter. Anim. Welf. 2006, 15, 371-377.

53. Carlstead, K.; Brown, J.L.; Strawn, W. Behavioral and physiological correlates of stress in laboratory cats. Appl. Anim. Behav. Sci. 1993, 38, 143-158. [CrossRef]

54. Gouveia, K.; Magalhães, A.; de Sousa, L. The behaviour of domestic cats in a shelter: Residence time, density and sex ratio. Appl. Anim. Behav. Sci. 2011, 130, 53-59. [CrossRef]

55. Stella, J.; Croney, C.; Buffington, T. Effects of stressors on the behavior and physiology of domestic cats. Appl. Anim. Behav. Sci. 2013, 143, 157-163. [CrossRef] [PubMed]

56. Delgado, M.M.; Munera, J.D.; Reevy, G.M. Human perceptions of coat color as an indicator of domestic cat personality. Anthrozoös 2012, 25, 427-440. [CrossRef]

57. Lepper, M.; Kass, P.H.; Hart, L.A. Prediction of adoption versus euthanasia among dogs and cats in a California animal shelter. J. Appl. Anim. Welf. Sci. 2002, 5, 29-42. [CrossRef] [PubMed]

58. Dybdall, K.; Strasser, R. Is there a bias against stray cats in shelters? People's perception of shelter cats and how it influences adoption time. Anthrozoös 2014, 27, 603-614. [CrossRef]

59. Sinn, L. Factors affecting the selection of cats by adopters. J. Vet. Behav. 2016, 14, 5-9. [CrossRef]

60. Weiss, E.; Miller, K.A.; Mohan-Gibbons, H.; Vela, C. Why did you choose this pet? Adopters and pet selection preferences in five animal shelters in the United States. Animals 2012, 2, 144-159. [CrossRef] [PubMed] 
61. Dybdall, K.; Strasser, R.; Katz, T. Behavioral differences between owner surrender and stray domestic cats after entering an animal shelter. Appl. Anim. Behav. Sci. 2007, 104, 85-94. [CrossRef]

62. Maddie's Institute. Foster Caregiver Involvement in Adoption. Community Pet Adoption Partnerships Survey Results; Maddie's Institute: Pleasanton, CA, USA, 2016. Available online: www.maddiesfund.org (accessed on 15 December 2016).

63. Lord, L.K.; Wittum, T.E.; Ferketich, A.K.; Funk, J.A.; Rajala-Schultz, P.; Kauffman, R.M. Demographic trends for animal care and control agencies in Ohio from 1996 to 2004. J. Am. Vet. Med. Assoc. 2006, 229, 48-54. [CrossRef] [PubMed]

64. Neidhart, L.; Boyd, R. Companion animal adoption study. J. Appl. Anim. Welf. Sci. 2002, 5, 175-192. [CrossRef] [PubMed]

65. Patronek, G.J.; Glickman, L.T.; Beck, A.M.; McCabe, G.P.; Ecker, C. Risk factors for relinquishment of cats to an animal shelter. J. Am. Vet. Med. Assoc. 1996, 209, 582-588. [PubMed]

66. Shore, E.R. Returning a recently adopted companion animal: Adopters' reasons for and reactions to the failed adoption experience. J. Appl. Anim. Welf. Sci. 2005, 8, 187-198. [CrossRef] [PubMed]

67. Podberscek, A.L. Illuminating issues of companion animal welfare through research into human-animal interactions. Anim. Welf. 1997, 6, 365-372.

(c) 2017 by the authors. Licensee MDPI, Basel, Switzerland. This article is an open access article distributed under the terms and conditions of the Creative Commons Attribution (CC BY) license (http:/ / creativecommons.org/licenses/by/4.0/). 